Combined ASRGL1 and p53 immunohistochemistry as an independent predictor of survival in endometrioid endometrial carcinoma

Huvila, Jutta

2018-04

Huvila , J , Laajala , T D , Edqvist , P-H , Mardinoglu , A, Talve , L , Ponten , F , Grenman , S , Carpen , O , Aittokallio , T \& Auranen , A 2018 , ' Combined ASRGL1 and p53 immunohistochemistry as an independent predictor of survival in endometrioid endometrial carcinoma ' , Gynecologic Oncology , vol. 149 , no. 1 , pp. 173-180 . https://doi.org/10.1016/j.ygyno.2018.02.016

http://hdl.handle.net/10138/301855

https://doi.org/10.1016/j.ygyno.2018.02.016

publishedVersion

Downloaded from Helda, University of Helsinki institutional repository.

This is an electronic reprint of the original article.

This reprint may differ from the original in pagination and typographic detail.

Please cite the original version. 


\title{
Combined ASRGL1 and p53 immunohistochemistry as an independent predictor of survival in endometrioid endometrial carcinoma
}

\author{
Jutta Huvila a,*,1, Teemu D. Laajala ${ }^{\mathrm{b}, \mathrm{c}, 1},{ }^{\mathrm{a}}$ Per-Henrik Edqvist ${ }^{\mathrm{d}}$, Adil Mardinoglu ${ }^{\text {e,f }}$, Lauri Talve ${ }^{\mathrm{a}}$, Fredrik Pontén ${ }^{\mathrm{d}}$, \\ Seija Grénman ${ }^{\mathrm{g}}$, Olli Carpén ${ }^{\mathrm{a}, \mathrm{h}, \mathrm{i}}$, Tero Aittokallio ${ }^{\mathrm{b}, \mathrm{c}}$, Annika Auranen $^{\mathrm{j}}$ \\ a Department of Pathology, University of Turku, Turku University Hospital, PL 52, 20520 Turku, Finland \\ b Department of Mathematics and Statistics, University of Turku, PL20, 00014 Helsinki, Finland \\ c Institute for Molecular Medicine Finland, FIMM, University of Helsinki, PL20, 00014 Helsinki, Finland \\ d Department of Immunology, Genetics and Pathology, Science for Life Laboratory, Uppsala University, BOX256, 75105 Uppsala, Sweden \\ e Science for Life Laboratory, KTH - Royal Institute of Technology, 10044 Stockholm, Sweden \\ ${ }^{\mathrm{f}}$ Department of Biology and Biological Engineering, Chalmers University of Technology, 41296 Gothenburg, Sweden \\ ${ }^{g}$ Department of Gynaecology and Obstetrics, University of Turku, Turku University Hospital, PL52, 20520 Turku, Finland \\ h Department of Pathology, University of Helsinki, Helsinki, Finland \\ i Finland HUSLAB, PL720, 00029, HUS, Finland \\ j Department of Gynaecology and Obstetrics, University of Tampere, Tampere University Hospital, PL2000, 33521 Tampere, Finland
}

\section{H I G H L I G H T S}

- ASRGL1 is a promising biomarker in endometrioid endometrial cancer.

- An immunopanel consisting of p53 and ASRGL1 is a useful tool in EEC risk assessment.

- Different EEC subgroups can be characterized using sophisticated statistical methods.

\section{A R T I C L E I N F O}

\section{Article history:}

Received 11 January 2018

Received in revised form 16 February 2018

Accepted 18 February 2018

Available online 2 March 2018

\section{Keywords:}

Endometrial cancer

Risk stratification

Prognostic

Modelling

ASRGL1

p53

\begin{abstract}
A B S T R A C T
Objective. In clinical practise, prognostication of endometrial cancer is based on clinicopathological risk factors. The use of immunohistochemistry-based markers as prognostic tools is generally not recommended and a systematic analysis of their utility as a panel is lacking. We evaluated whether an immunohistochemical marker panel could reliably assess endometrioid endometrial cancer (EEC) outcome independent of clinicopathological information.

Methods. A cohort of 306 EEC specimens was profiled using tissue microarray (TMA). Cost- and time-efficient immunohistochemical analysis of well-established tissue biomarkers (ER, PR, HER2, Ki-67, MLH1 and p53) and two new biomarkers (L1CAM and ASRGL1) was carried out. Statistical modelling with embedded variable selection was applied on the staining results to identify minimal prognostic panels with maximal prognostic accuracy without compromising generalizability.

Results. A panel including p53 and ASRGL1 immunohistochemistry was identified as the most accurate predictor of relapse-free and disease-specific survival. Within this panel, patients were allocated into high- (5.9\%), intermediate- $(29.5 \%)$ and low- $(64.6 \%)$ risk groups where high-risk patients had a 30 -fold risk $(P<0.001)$ of dying of EEC compared to the low-risk group.

Conclusions. P53 and ASRGL1 immunoprofiling stratifies EEC patients into three risk groups with significantly different outcomes. This simple and easily applicable panel could provide a useful tool in EEC risk stratification and guiding the allocation of treatment modalities.
\end{abstract}

(C) 2018 Elsevier Inc. All rights reserved.
* Corresponding author at: Department of Pathology, University of Turku, Kiinamyllynkatu 8-10, FIN-20520 Turku, Finland.

E-mail address: jutta.huvila@utu.fi (J. Huvila).

${ }^{1}$ Equal contribution

\section{Introduction}

Endometrial cancer (EC) is the most common gynaecologic cancer in developed countries. Approximately $80 \%$ of EC cases are of endometrioid (EEC) type. The majority of EEC cases are detected at an 
early stage (Figo stages I and II) when the prognosis is generally favourable. Still, over $10 \%$ of early stage EEC cancers relapse $[1,2]$. Currently, we are unable to assess accurately the outcome of early EEC patients. This either leads to unnecessary follow-up for the majority of patients, or to suboptimal treatment of the patients who eventually suffer from relapse. Following the principles of disease stratification as a step towards personalized treatment of EEC, biomarkers are needed to identify patient subgroups that would most benefit from additional adjuvant therapy. A number of tissue biomarkers have been introduced for EEC prognostication [3,4]. However, the integration of biomarkers into clinical guidelines has been slow.

In this study, our aim was to identify a prognostic panel of biomarkers based solely on immunohistochemical stainings, accompanied by sophisticated statistical modelling. More specifically, we wanted to test whether prognostication of EEC would be feasible using only tissue-derived parameters. For this purpose, we chose a set of wellestablished tissue markers that are known to have a prognostic value in EEC. These include ER and PR, whose prognostic role has been confirmed in several studies [5,6]. P53 was included, as its independent prognostic role in EC has repeatedly been shown [7-9]. Additional biomarkers included Ki-67, MLH1 and HER-2 [10-12]. Finally, two recently characterized promising biomarkers were included: L1-cell adhesion molecule (L1CAM) and L-asparaginase like 1 (ASRGL1). The prognostic value of L1CAM has been shown in various settings, both in type I and II EC [2,13-18] and it has attained an established role in EC biomarker panels both in retrospective and prospective studies. ASRGL1 is a novel biomarker candidate, which we recently demonstrated to have an independent prognostic value in two independent EEC cohorts [19] and the prognostic role of ASRGL1 was confirmed in a recent study [20]. Additionally, the ASRGL1 gene has been identified as an important hub gene when comparing most differentially expressed genes between EEC and non-endometrioid carcinoma [21].

Although disease stratification by integration of different "omics" data is a promising future approach [22], both technological and financial constraints limit these methods to a few centres. For routine clinical diagnostics, there is still a dire need for robust, low-cost and widely applicable prognostic tools. In this study, sophisticated statistical modelling techniques were used to identify minimal panels of immunohistochemical markers that are capable of providing maximally predictive and practical tools for comprehensive prediction and modelling of EEC patient characteristics. Our main aim was to construct two separate panels; one useful in a pre-operative setting, which would predict clinicopathological risk-factors and aid in surgical treatment planning, and another to be used in a postoperative setting to identify patients who are at risk of disease relapse or death due to the disease.

\section{Material and methods}

\subsection{Patient characteristics}

During the years 2001-2007, 327 women with endometrial cancer were operated in Turku University Hospital. Both full patient records and paraffin-embedded material were available. From this cohort, we excluded 14 patients with a non-endometrioid or mixed-type histology, four patients who received any form of pre-operative treatment and three due to unsatisfactory tissue material. The remaining 306 EEC patients were included in this study. All the patients were restaged (AA, $\mathrm{JH}$ ) based on patient record information in accordance with the FIGO 2009 staging guidelines [23]. The study was approved by the Ethical Committee of the Southwestern Finland Hospital District and the Finnish National Authority for Medicolegal Affairs.

The histopathology of each tumor was re-classified by two expert gynaecopathologists (LT, OC) according to the FIGO guidelines [23,24]. Demographic, clinical, and pathologic information and follow-up data for relapse or death was obtained from the hospital records. The patients were followed until September 2014 or death. The survival data were obtained from the hospital records and the Population Registry, and the cause of death from Statistics Finland. Disease relapse and death due to disease were registered as the end-point events for postoperative modelling. No patients were lost to follow-up.

The clinical and pathologic features of the 306 EEC patients included in this study are summarized in Table $1 \mathrm{~A}$. All patients were surgically treated; $74 \%$ underwent surgical staging, of which $58.2 \%$ underwent pelvic and $15.4 \%$ both pelvic and para-aortic lymphadenectomy. Adjuvant therapy was primarily given according to the hospital guidelines, provided that there was no patient-related impairment.

During a median follow-up of 7.2 years (range $0.15-13.02$ ), 40 (13.1\%) of the patients experienced a relapse. The majority of these (87.5\%) were distant relapses. During a median 7.4 years follow-up, $10.1 \%$ died of EEC.

\subsection{Tissue microarrays (TMAs) and immunohistochemistry (IHC)}

Generation of TMAs, basic IHC techniques, and slide scanning were performed as previously described $[19,25]$ at the Swedish Science for Life Laboratory (SciLifeLab) Tissue Profiling Facility at Uppsala University (Sweden), in accordance with the standards used in The Human Protein Atlas project (www.proteinatlas.org) [26]. The study was performed in accordance with recommended biomarker reporting guidelines (REMARK). Detailed information on the antibodies used in this study is presented in supplementary data.

The immunohistochemically stained slides were scanned and independently evaluated by two pathologists ( $\mathrm{OC}$ and $\mathrm{JH}$ ). Both tissue cores for each case were analysed and an average was determined and used for further analysis. If staining result was assessed as a continuous variable, an average of the two cores was calculated. In cases with discrepancies in evaluation, the slides were re-evaluated until an agreement was reached.

Immunoreactivity for ER, PR, ASRGL1 and Ki-67 was quantitatively scored based on positive staining of tumor cells. The frequency was assessed semi-quantitatively in 6 classes $(0 \%$; $1-10 \%$; $11-25 \%$; 25-50\%; 51-75\% and $>75 \%$ thresholds). P53 staining was considered aberrant if cancerous cells were completely negative, or if moderateto-strong nuclear staining was present in over $75 \%$ of the tumor cells. HER-2 staining was considered positive if membranous staining in $>10 \%$ of the tumor cells of strong intensity were present. MLH1 was considered negative if there was no evident staining in the cancer cells but stromal cells showed positive staining. L1CAM was considered negative if $<10 \%$ of tumor cells were positive, as on the threshold if approximately $10 \%$ of cells were negative, and as positive if $>10 \%$ were positive

Table 1

Clinicopathological characteristics of the presented 306 EEC cases (A) and their association with risk assessing immunoprofile (B, $n=305)$.

\begin{tabular}{|c|c|c|c|c|c|}
\hline \multirow[t]{2}{*}{ A } & & & \multicolumn{3}{|l|}{ B } \\
\hline & & & Low-risk ${ }^{\mathrm{b}}$ & Intermediate-risk $^{\mathrm{c}}$ & High-risk $^{\mathrm{d}}$ \\
\hline Age $^{a}$ & & $66(59-73)$ & $66(59-73)$ & $66(58-74)$ & $70(65-76)$ \\
\hline \multirow[t]{4}{*}{ Figo stage } & I & $247(80.7 \%)$ & $171(69.5 \%)$ & $64(26.0 \%)$ & $11(4.5 \%)$ \\
\hline & II & $9(2.9 \%)$ & $7(77.8 \%)$ & $1(11.1 \%)$ & $1(11.1 \%)$ \\
\hline & III & $42(13.7 \%)$ & $16(38.1 \%)$ & $23(54.8 \%)$ & $3(7.1 \%)$ \\
\hline & IV & $8(2.6 \%)$ & $3(37.5 \%)$ & $2(25.0 \%)$ & $3(37.5 \%)$ \\
\hline \multirow[t]{3}{*}{ Grade } & 1 & $166(54.2 \%)$ & $131(78.9 \%)$ & $32(19.3 \%)$ & $3(1.8 \%)$ \\
\hline & 2 & $87(28.4 \%)$ & $52(59.8 \%)$ & $30(34.5 \%)$ & $5(5.7 \%)$ \\
\hline & 3 & $53(17.3 \%)$ & $14(26.9 \%)$ & $28(53.8 \%)$ & $10(19.2 \%)$ \\
\hline \multirow[t]{2}{*}{ MI } & $<50 \%$ & $206(67.3 \%)$ & $144(70.2 \%)$ & $52(25.4 \%)$ & $9(4.4 \%)$ \\
\hline & $\geq 50 \%$ & $100(32.7 \%)$ & $53(53.0 \%)$ & $38(38.0 \%)$ & $9(9.0 \%)$ \\
\hline \multirow[t]{3}{*}{ LVI } & No & $203(66.3 \%)$ & $131(64.9 \%)$ & $59(29.2 \%)$ & $12(5.9 \%)$ \\
\hline & Yes & $37(12.1 \%)$ & $15(40.5 \%)$ & $18(48.6 \%)$ & $4(10.8 \%)$ \\
\hline & Missing & $66(21.6 \%)$ & & & \\
\hline
\end{tabular}

$\mathrm{N}(\%)$

a Median (IQR).

b Low-risk: p53 wild-type, ASRGL1 > 75\%.

c Intermediate-risk: p53 wild-type, ASRGL1 $\leq 75 \%$ or p53 aberrant and ASRGL1 $>75 \%$.

d High-risk: p53 aberrant, ASRGL1 $\leq 75 \%$. 
[2]. Positive tumor samples, non-malignant areas and stromal tissue in tumor specimens served as controls.

For prognostic modelling, ER, PR and ASRGL1 were classified using conventional frequency thresholds in order to facilitate the clinical use of the potential immunopanel. The threshold approach was also applied to minimize potential methodological issues, such as the reproducibility of intensity values across laboratories, and thereby to increase the comparability and generalizability of the models to different clinical setups [27].

\subsection{Statistical modelling}

The prognostic variables for the predictive modelling included age, p53 status, HER-2 overexpression, MLH1 positivity, L1CAM status, and the frequencies of PR, ER, Ki67 and ASRGL1. The immunostaining frequencies were systematically tested with varying cutoffs, and incorporated as binary indicators in the regression model. In post-operative modelling, radiotherapy (pelvic and/or brachytherapy), chemotherapy and operational treatment were included to adjust for potential confounding effects. Potential confounder coefficients were omitted from the final model. LASSO logistic regression [28] and its Cox variant [29] were utilized for predictive modelling based on the pre- and post-operative panels, respectively. A sequence of lambda LASSO parameters was tested using 10-fold cross-validation (CV) to optimize ROC-AUC and c-index metrics in the logistic and Cox regression, respectively. The final models were fitted using conservative lambda value within one standard error of the CV-optimum, which resulted in the proposed optimal set of predictive markers as well as their specific thresholds by the feature selection embedded in LASSO regression covering all the potential marker candidates. The $P$-values for the identified pre-operative panels were computed using the Fisher's exact test. Statistical testing of the post-operative risk groups was performed using conventional Cox proportional hazards. All statistical tests were two-sided.

The heatmap clustering of the immunohistological variables was conducted using complete linkage with the Euclidean distance. The cut-offs were derived from the optimized LASSO models. The R software (version 3.3.1) was utilized in all the statistical analyses extended by R-packages glmnet (version 2.0-5) and hamlet (version 0.9 .5 ) (both publicly available in the CRAN repository). The R-code for reproducing data analysis and modelling is provided in the Supplementary Material.

\subsection{ASRGL1 gene expression}

To correlate ASRGL1 gene expression with outcome, endometrial cancer patient samples $(n=541)$ from The Cancer Genome Atlas (TCGA) project were used. Both transcriptomics data (RNA-Seq) and clinical information was collected from the Genomic Data Commons (GDC) (https://gdc-portal.nci.nih.gov/). Transcriptomics data was retrieved as FPKM (fragments per kilobase of exon per million mapped reads) values from tumor tissues representing endometrial cancer and clinical data included overall survival time for corresponding patients. In the analysis, we excluded genes with low expression, i.e., those with a median expression among samples less than one. A cut-off value of 1 FPKM was used as a detection limit.

Based on the ASRGL1 FPKM values, patients were classified into two groups The prognosis of each group of patients was examined by Kaplan-Meier survival estimators, and the survival outcomes of the two groups were compared by log-rank tests. To choose the optimal FPKM cut-off for stratifying the patients into two different prognostic groups, the most significant $P$-value within the 20th to 80th percentile was used and the cut -off for low and high ASRGL1 expression groups was set at 55.3 FPKM.

\section{Results}

Two different risk stratification strategies were assessed; one for post-operative risk stratification and the other for pre-operative risk assessment. The post-operative prognostic model identified the combination of p53 and ASRGL1 (cut-off 75\%) staining as the best predictor of both disease-free survival (DFS) and disease-specific survival (DSS). Based on the model-identified prognostic panel, the patients were grouped into three risk categories: low-risk (p53 wild type, ASRGL1 $>75 \%$ ), intermediate-risk (p53 wild type, ASRGL1 $\leq 75 \%$ or p53 aberrant, ASRGL1 > 75\%) and high-risk (p53 aberrant, ASRGL1 $\leq 75 \%$ ). Of all 306 EEC cases, $64.6 \%$ belonged to the low-risk group, $29.5 \%$ to the intermediate-risk group and 5.9\% to the high-risk group. The survival rates of these categories are presented in Fig. 1A. In the high-risk group, $61.1 \%(11 / 18)$ patients died from disease, compared to $15.6 \%$ (14/90) in the intermediate-risk group and 3.0\% (6/197) of patients in the low-risk group. Patient records of the low-risk patients that died of disease were revisited. Of these six patients, three had advanced, stage III or IV disease. Further, a patient died of an EEC treatment related cause while another underwent only vaginal hysterectomy due to uterine prolapse, relapsed and died of disease after 5.5 years of EEC despite compensatory radiation therapy. For the single remaining case no risk factor could be identified.

When compared to the low-risk group patients, patients considered to have an intermediate-risk disease had a 5.8-fold risk [HR 5.85(CI 2.22-15.33), $P<0.001]$ whereas the high-risk disease cases had a $30-$ fold risk [HR 30.1(CI 10.93-83.14), $P<0.001$ ] of dying from disease [HR 30.1(CI 10.93-83.14), $P<0.001$, Cox regression model]. The association between the risk classes, determined by ASRGL1 and p53 expression, and clinicopathological factors is presented in Table 1B. Stage (I-II/III-IV), grade and age where entered in a Cox regression model (backward stepwise likelihood ratio) together with risk-class data. Stage and risk-class where included in the final model where stage had a HR of 5.31 (CI 2.53-11.10, $P<0.001)$ and intermediaterisk and high-risk classes a HR of 3.93 (CI 1.46-10.57, $P<0.007$ ) and HR 20.38 (CI 7.19-57.72, $P<0.001$ ), respectively.

Based on the statistical modelling, cut-off values were set for each marker, and these values were used to categorize the EECs into groups. The cut-offs and numbers of cases within each category are shown in Table 2. The IHC results were clustered together with the clinicopathological variables as a dendrogram and a heatmap was produced to illustrate the relationship between the different markers (Fig. 2), with the visualized marker thresholds chosen according to the identified optimal models. As shown in the heatmap, hormonal receptors (ER, PR) were correlated with ASRGL1 (pairwise Spearman rho correlations: ER and PR: 0.6527; ASRGL1 and ER: 0.4218; and ASRGL1 and PR: 0.5339, with all pairwise correlations highly statistically significant $P<0.001$ ). The cases with sparse or negative staining for these biomarkers were clustered together. Also the clinical end-points, death from disease and disease relapse, as well as cases with high-grade and advanced disease were classified into the ER/PR/ASRGL1 negative clusters indicating a clinical significance of the these markers.

In the current EEC cohort, a statistically significant co-expression was found between the p53 status and L1CAM stainings (Fisher's exact test, $P<0.001$, Fig. 2). Of the p53 aberrant cases, 13 (36.1\%) were L1CAM positive, and of the 27 L1CAM positive cases 13 (48.1\%) were p53 aberrant. Additionally, 8 (47.1\%) of the 17 cases with highrisk immunoprofile were L1CAM positive. The correlation between the L1CAM and p53 expression and the disease-specific and recurrencefree survival is presented in Fig. 1B. According to our analyses, p53 aberrant tumours had a significantly adverse prognosis regardless of the L1CAM status. However, the overlap of the two markers was significant.

Moreover, we searched for a potential pre-operative panel for deep myometrial invasion (MI), presence of lymphovascular invasion (LVI) and advanced, FIGO stage III-IV disease. Three panels consisting of ER, PR and/or ASRGL1 with different threshold values were identified 
A

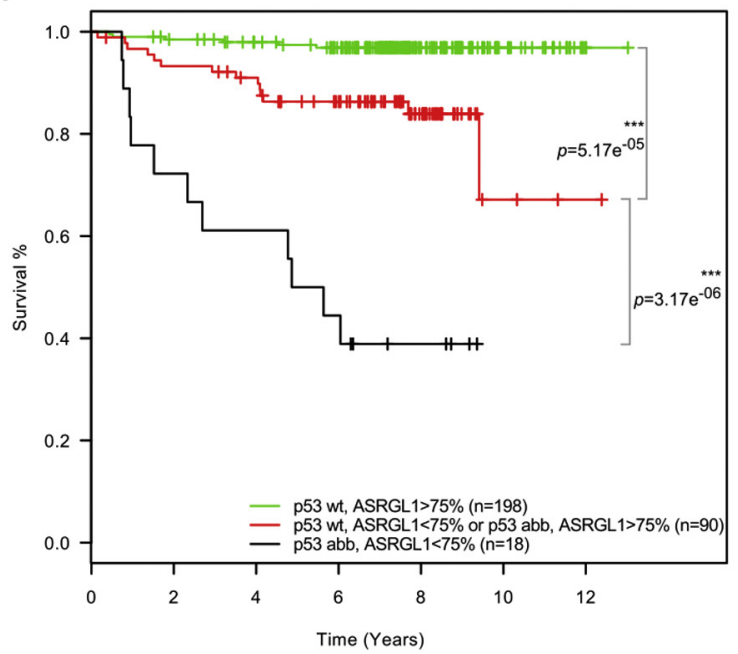

B

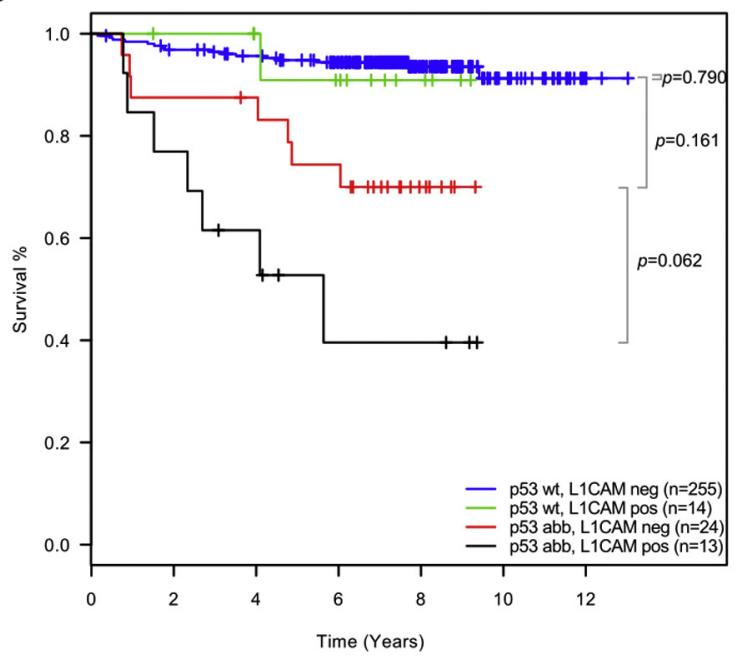

Recurrence

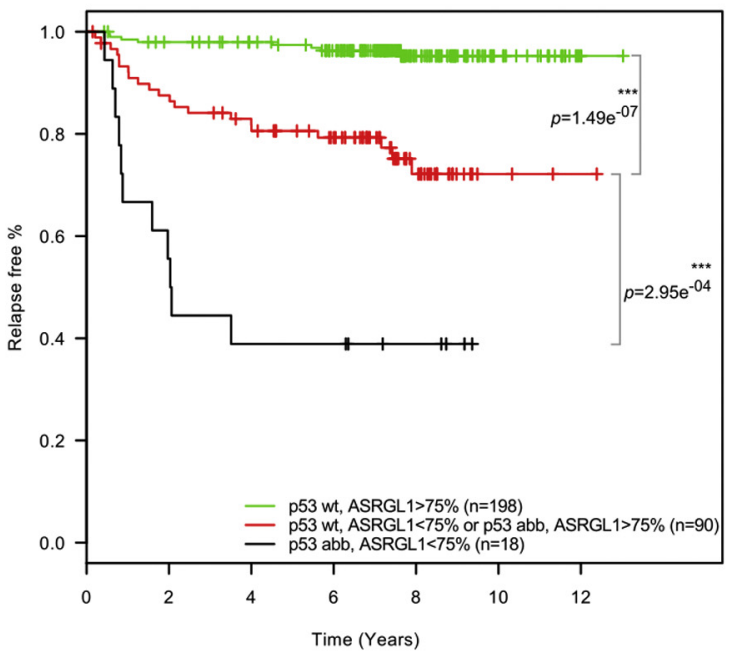

Recurrence

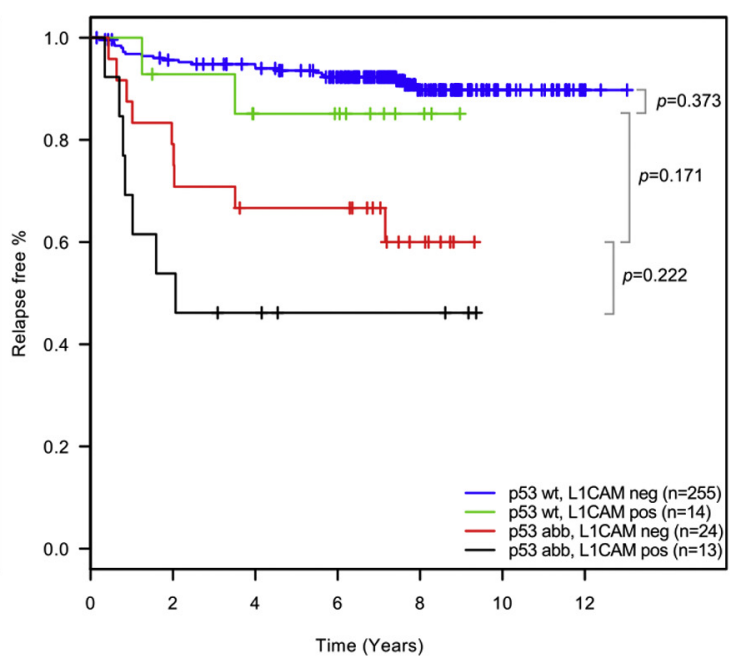

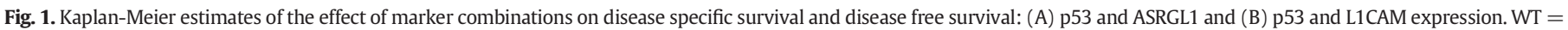
wild type, $\mathrm{AB}=$ aberrant $\mathrm{p} 53$ expression. ${ }^{* * *} \mathrm{P}<0.001$.

with the statistical modelling (Table 3). ER (cut-off 50\%) was the best predictor of MI. For LVI, ER (cut-off 1\%) combined with PR (cut-off $25 \%)$ provided the best panel for prediction. A large majority, 149 (93\%), of the hormone receptor positive cases did not present with LVI. Of the LVI-positive EECs, 26 (70\%) were negative for ER and/or PR. Finally, the combination of PR (cut-off 10\%) and ASRGL1 (cut-offs 25\% and 75\%) was the best predictor for advanced disease (FIGO stage III-IV).

Finally, to further validate our results also on the mRNA level in an independent cohort, we utilized gene expression data from the publicly available TCGA data set [30]. To study the association between ASRGL1 expression and outcome for patients with endometrial cancer, ASRGL1 gene expression (mRNA) was assessed from 541 tumours and the cut -off for low and high ASRGL1 expression groups was set at 55.3 FPKM. A Kaplan-Meier analysis based on this cut-off showed that patients with tumours showing low expression of ASRGL1 have a significantly $(P<0.001)$ shorter overall survival rate as compared to patients with high ASRGL1 expression (Fig. 3).

\section{Discussion}

There is a profound need for improved risk assessment methods for patients with EEC. The existing risk stratifications guiding surgical treatment and adjuvant therapy allocation consist of clinicopathological variables. These variables i.e. stage, histology, grade, myometrial invasion and lymphovascular invasion often associate with disease progression and survival, but may not truly reflect tumor biology [31]. Moreover, the reproducibility of FIGO grading has been unsatisfactory $[11,24]$, and significant discrepancy rates have been reported [1]. Tumor grade can be assessed on pre-operative samples, but the consistency between pre- and post-operative findings can be modest [32]. The assessment of MI and LVI can only be done on the surgical specimen, and both of these events are dependent on the representative sampling of tumor specimen and prone to errors such as pseudoinvasion [33]. None of the current risk classifications provide high accuracy in stratifying the risk of recurrence or nodal metastasis [34]. Additionally, discrepancies between the pre- and postoperative risk assessment have been reported [35], emphasizing the need for improved diagnostic tools to be developed using robust statistical methods.

We show that a panel of two IHC markers only, p53 and ASRGL1, could be a useful tool in risk assessment of EEC. With p53 and ASRGL1 immunohistochemistry, patients can be categorized into three risk classes in each of which the outcome is significantly different (Fig. 1). Patients with aberrant p53 and low ASRGL1 $(\leq 75 \%)$ expression are at the highest risk for relapse or disease-related death. The inclusion of p53 in the panel is not surprising as its prognostic role in EEC has been shown earlier [7-9]. Although aberrant p53 is typically associated 
Table 2

The distribution of immunohistochemical staining results.

\begin{tabular}{|c|c|}
\hline ER & $\mathrm{N}(\%)$ \\
\hline$<1 \%$ & $39(12.7 \%)$ \\
\hline $1-50 \%$ & 79 (25.8\%) \\
\hline$>50 \%$ & $188(61.4 \%)$ \\
\hline \multicolumn{2}{|l|}{$\mathrm{PR}^{*}$} \\
\hline$<10 \%$ & $64(21.0 \%)$ \\
\hline $10-25 \%$ & $18(5.9 \%)$ \\
\hline$>25 \%$ & $223(73.1 \%)$ \\
\hline \multicolumn{2}{|l|}{ ASRGL1* } \\
\hline$<25 \%$ & $40(13.1 \%)$ \\
\hline $25-75 \%$ & 49 (16.0\%) \\
\hline$>75 \%$ & $216(70.8 \%)$ \\
\hline \multicolumn{2}{|l|}{ p53 } \\
\hline Wild type & 269 (87.9\%) \\
\hline Aberrant & 37 (12.1\%) \\
\hline \multicolumn{2}{|l|}{ L1CAM* $^{*}$} \\
\hline Negative & $278(91.1 \%)$ \\
\hline Threshold & $7(2.3 \%)$ \\
\hline Positive & $20(6.6 \%)$ \\
\hline \multicolumn{2}{|l|}{ 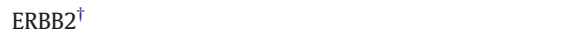 } \\
\hline Normal & 293 (98.7\%) \\
\hline Overexpressed & $4(1.3 \%)$ \\
\hline \multicolumn{2}{|l|}{ MLH1 } \\
\hline Negative & 91 (29.7\%) \\
\hline Positive & $215(70.3 \%)$ \\
\hline \multicolumn{2}{|l|}{ Ki-67 } \\
\hline$<25 \%$ & 137 (44.8\%) \\
\hline $25-75 \%$ & $139(45.4 \%)$ \\
\hline$>75 \%$ & 30 (9.8\%) \\
\hline
\end{tabular}

with non-endometrioid serous carcinoma, a fraction of EECs are also characterized by mutant p53 [22]. Somewhat surprisingly, L1CAM was not included in the prognostic panel, despite studies showing its prognostic value in EC $[14,17,36]$. The most likely explanation for this observation is, that L1CAM expression has been found to correlate with aberrant p53 and to be related to non-endometrioid morphology $[14,17,36]$. The penalized regression methodology aims to find a minimal set of explaining variables thus leading to the inclusion of only a single variable of a set of highly correlated markers, and eludes markers that do not add to the prognostic value of the panel. Thus, in the present study the LASSO regression model p53 was superior to L1CAM. A small proportion of the cases in this study presented a endometrioid histology but were, however, both $\mathrm{p} 53$ aberrant and L1CAM positive indicating an immunohistochemical expression typical to serous carcinoma. Distinguishing EEC from serous carcinoma from H\&E slides is often rather straightforward, however, the morphological subclassification might fail to identify a proportion of non-endometrioid cases [37] and the reproducibility of histiotype is problematic even in the most experienced hands [38].

ASRGL1 is a novel biomarker candidate, which has demonstrated to have an independent prognostic value in EEC $[19,20]$. In this study, we show in an independent cohort that in addition to reduced ASRGL1 protein expression, also low ASRGL1 gene expression is associated with adverse prognosis, which further strengthens the prognostic role of ASRGL1 expression in endometrial cancer cells. Additional confirmatory evidence for the role of ASRGL1 in EC has come from a recent gene regulatory network analysis, which identified ASRGL1 as an important hub gene when comparing most differentially expressed genes between EEC and non-endometrioid carcinoma [21]. Although ASRGL1 was not associated with EEC specific survival in the aforementioned study, the results emphasize the importance of ASRGL1 in EEC.

ASRGL1 is demonstrated to degrade both L-asparagine and isoaspartyl peptides in vitro [39] indicating that the loss of ASRGL1 could lead to elevated asparagine levels. In a recent study on breast cancer, the authors show that asparagine synthetase expression in a primary tumor correlated with later metastatic relapse suggesting that the bioavailability of asparagine strongly influences metastatic potential [40]. Further on, altering asparagine availability had an influence on invasive potential, thought to relate to the effect on proteins that promote the epithelial-to-mesenchymal transition [40]. The authors also analysed the TCGA Pan-Cancer expression data of asparaginase synthetase (ASNS) and showed that endometrial cancer survival was better in the ASNS low -group, further emphasizing the importance of asparagine in endometrial cancer [40]. The metastatic potential of ASRGL1 depleted EC is supported by the finding made by Fonnes et al., who showed that $77 \%$ of metastatic lymph nodes were ASRGL1 negative and that many ASRGL1 high primary tumours presented with ASRGL1 low metastasis [20].

The L-Asparaginase has long been used to treat acute lymphoblastic leukaemia but has not yet been as successful in solid tumours. Still, high asparagine levels in EC - either due to high asparagine synthetase or low asparaginase levels - could potentially be targeted with L-asparaginase treatment.
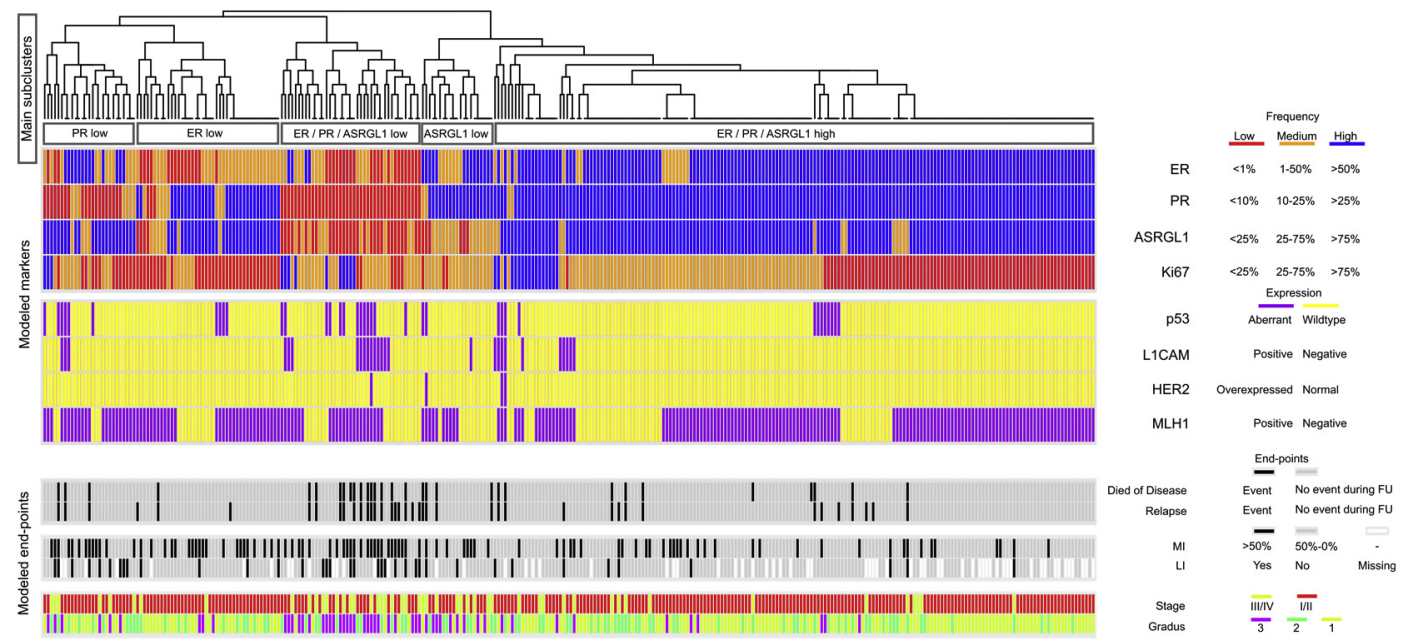

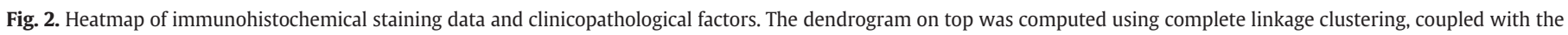
Euclidean distance. 
Table 3

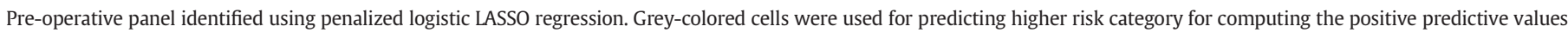
(PPV) and negative predictive values (NPV).

A) Myometrium invasion
\begin{tabular}{|l|l|l|}
\hline & Depth of myometrium invasion \\
\hline ER Frequency & $<50 \%$ & $>50 \%$ \\
\hline$>50 \%$ & $148(78.7 \%)$ & $40(21.3 \%)$ \\
\hline$<50 \%$ & $58(49.2 \%)$ & $60(50.8 \%)$ \\
\hline
\end{tabular}

Cells indicate number of samples (proportion).

$P<0.001$, Fisher's exact test; PPV: $50.9 \%$, NPV: $78.7 \%$.

B) Lymphovascular invasion ${ }^{*}$

\begin{tabular}{|l|l|l|l|l|}
\hline \multirow{2}{*}{} & \multicolumn{4}{|l|}{} \\
\cline { 2 - 5 } & No & \multicolumn{2}{l|}{ Yes } \\
\hline PR Frequency & $>25 \%$ & $34 \%$ & $>25 \%$ & $<25 \%$ \\
\hline ER Freq. $>1 \%$ & $149(72.3 \%)$ & $34(16.5 \%)$ & $11(5.3 \%)$ & $12(5.8 \%)$ \\
\hline ER Freq. $<1 \%$ & $8(24.2 \%)$ & $11(33.3 \%)$ & $2(6.1 \%)$ & $12(36.4 \%)$ \\
\hline
\end{tabular}

${ }^{*} 67$ missing values $(28 \%)$.

$P<0.001$, Fisher's exact test; PPV: $32.9 \%$, NPV: $93.1 \%$.

C) Stage I/II versus III/IV

\begin{tabular}{|c|c|c|c|c|}
\hline & \multicolumn{4}{|l|}{ Stage $^{*}$} \\
\hline & \multicolumn{2}{|l|}{$\mathrm{I} / \mathrm{II}$} & \multicolumn{2}{|l|}{ III/IV } \\
\hline PR Frequency & $>10 \%$ & $<10 \%$ & $>10 \%$ & $<10 \%$ \\
\hline ASRGL1 $>75 \%$ & $184(85.2)$ & $11(5.1 \%)$ & $16(7.4 \%)$ & $5(2.3 \%)$ \\
\hline ASRGL1 25-75\% & $27(49.1 \%)$ & $14(25.5 \%)$ & $8(14.5 \%)$ & $6(10.9 \%)$ \\
\hline ASRGL $1<25 \%$ & $2(6.1 \%)$ & $16(48.5 \%)$ & $4(12.1 \%)$ & $11(33.3 \%)$ \\
\hline
\end{tabular}

The model predicting deep MI, LVI and advanced disease suggested that ER, PR and ASRGL1 could be useful markers in such risk assessment. No single model or panel to predict the high-risk cases could be identified, which suggest that different mechanisms may lead to similar patterns of disease spread and invasion. Further complexity stems from the uncertainty in the evaluation of these clinicopathological variables. However, our results further confirm the role of ER, PR and ASRGL1 in preoperative risk assessment of EEC.

There is currently a relatively large variation in the cut-off values used to determine immunohistochemical staining results in EEC and one of our aims was to search for clinically relevant cut-off values for the immunomarkers. Our data suggested that the currently used definition of aberrant p53 expression and ASRGL1 positivity in over $75 \%$ of cancer cells are good predictors of survival in EEC. The widely studied biomarkers in ER and PR still lack established cut-off values, and in many cases the used cut-off values are the same used in breast cancer diagnostics, typically $>10 \%$ and $>1 \%$. In our study, no single cut-off could be determined and further work is needed to determine optimal cut-offs.

The Cancer Genome Atlas (TCGA) data highlighted the endometrial cancer heterogeneity, especially in high-grade carcinomas in EC $[22,41]$. Based on the TCGA data, a new classification tool, ProMisE, was developed, using IHC determination of DNA mismatch repair genes and p53, and an analysis of POLE mutation profile [42] to identify those risk groups presented in the TCGA study. The immunopanel developed in the present study shares some elements (p53, MLH1) with the ProMisE classification tool. Our main aim was not to categorize patients but rather to evaluate the prognostic capacity of an immunopanel in risk assessment of EC, therefore complementing the prior results from TCGA.

The strengths of our study include a well-described, unselected patient cohort, with complete clinical follow-up data. We used universal, easily assessable and usable immunohistochemical stainings, which have good repeatability across different laboratories. Our study material was selected to consist only of morphologically defined EEC, however the immunohistological results indicate that a portion of these could represent serous- or serous like carcinomas. As stated above, subclassification based on purely morphological features might fail to identify a proportion of non-endometrioid cases highlighting the need for biomarkers. Another major strength of the current study is the systematic statistical modelling and variable selection technique applied in both the pre- and post-operative panels, which not only identified the maximally predictive panels of markers, but also recognized their clinically relevant cut-off thresholds without á priori assumptions. One weakness of this study is that the presented immunopanel remains yet to be validated in a separate cohort. Additionally, 215 of the extended cohort with 306 patients were readily included in the original ASRGL1 study [19], and there is a possibility of slight overfitting of ASRGL1 in this material due to re-modelling parts of the data. 
Died of Disease (ASRGL1)

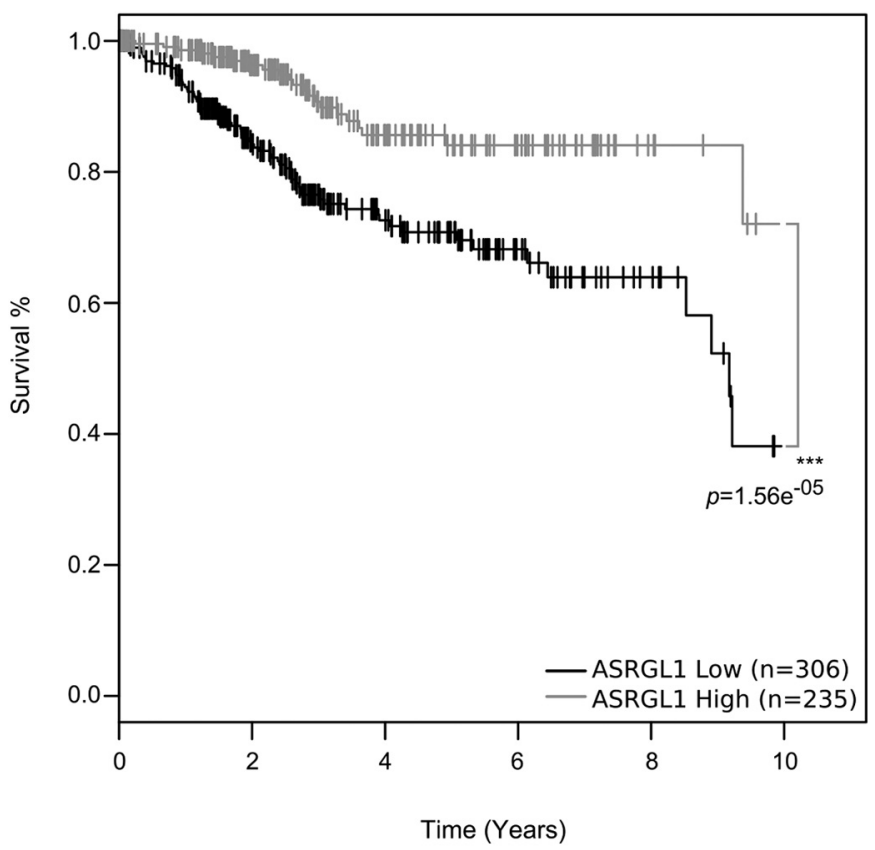

Fig. 3. Kaplan-Meier plot based ASRGL1 mRNA expression shows that the patient group ( $n$ $=306$ ) with relative low $(<55.3$ FPKM) expression levels of ASRGL1 has a decreased overall survival rate compared to the patient group $(n=235)$ with a relative high level (>55.3 FPKM) of ASRGL1 expression.

Our results emphasize the prognostic role of p53 expression in EEC. Additionally, our results support the independent prognostic role of the novel biomarker ASRGL1 and highlight its prognostic value as compared to several established biomarkers. Further studies are needed to confirm the prognostic immunopanel presented in this study and to verify the optimal thresholds for EEC prognosis, preferably in a prospective setting. Also, translational studies are required to understand the biological implications between reduced ASRGL1 expression and EEC aggressiveness.

In conclusion, a simple and affordable immunohistochemistry panel consisting of p53 and ASRGL1 provides important information for EEC risk stratification. Such stratification could help to modify follow-up strategies and reduce unnecessary visits and health-care costs without compromising outcome results.

Supplementary data to this article can be found online at https://doi. org/10.1016/j.ygyno.2018.02.016.

\section{Funding}

This work was supported by University of Turku Doctoral Program of Clinical Investigations, Regional Fund of Finland Proper of the Finnish Cultural Foundation (grant 85121904), Medical Research Fund (EVO) of Turku University Hospital [JH]; Academy of Finland (grants 292611, 269862, 272437, 279163, 295504), National Cancer Institute (16X064), Cancer Society of Finland [TA] and by grants from the Knut and Alice Wallenberg Foundation (FP, AM, PHE) and the Swedish Cancer foundation (FP).

\section{Disclosure/conflict of interest}

As a conflict of interest, Atlas Antibodies AB has filed a patent (ASRGL1 in endometrial cancer), where FP and PHE are listed as inventors. Other authors have no conflict of interest to declare.

\section{References}

[1] C.L. Creutzberg, W.L. van Putten, P.C. Koper, M.L. Lybeert, J.J. Jobsen, C.C. WárlámRodenhuis, et al., Surgery and postoperative radiotherapy versus surgery alone for patients with stage-1 endometrial carcinoma: multicentre randomised trial. PORTEC study group. Post operative radiation therapy in endometrial carcinoma, Lancet 355 (2000) 1404-1411.

[2] A.G. Zeimet, D. Reimer, M. Huszar, B. Winterhoff, U. Puistola, S.A. Azim, et al., L1CAM in early-stage type I endometrial cancer: results of a large multicenter evaluation, J. Natl. Cancer Inst. 105 (2013) 1142-1150.

[3] R. Murali, R.A. Soslow, B. Weigelt, Classification of endometrial carcinoma: more than two types, Lancet Oncol. 15 (2014) e268-78.

[4] H.M. Werner, H.B. Salvesen, Current status of molecular biomarkers in endometrial cancer, Curr. Oncol. Rep. 16 (2014) 403.

[5] V. Jongen, J. Briet, R. de Jong, K. ten Hoor, M. Boezen, A. van der Zee, et al., Expression of estrogen receptor-alpha and -beta and progesterone receptor- $A$ and -B in a large cohort of patients with endometrioid endometrial cancer, Gynecol. Oncol. 112 (2009) 537-542.

[6] M. Köbel, E.G. Atenafu, P.F. Rambau, S.E. Ferguson, G.S. Nelson, T.C. Ho, et al., Progesterone receptor expression is associated with longer overall survival within highgrade histotypes of endometrial carcinoma: a Canadian high risk endometrial cancer consortium (CHREC) study, Gynecol. Oncol. 141 (2016) 559-563.

[7] K. Garg, M.M.J. Leitao, C.A. Wynveen, G.L. Sica, J. Shia, W. Shi, et al., p53 overexpression in morphologically ambiguous endometrial carcinomas correlates with adverse clinical outcomes, Mod. Pathol. 23 (2010) 80-92.

[8] R.A. Nout, T. Bosse, C.L. Creutzberg, I.M. Jürgenliemk-Schulz, J.J. Jobsen, L.C. Lutgens, et al., Improved risk assessment of endometrial cancer by combined analysis of MSI, PI3K-AKT, Wnt/ß-catenin and P53 pathway activation, Gynecol. Oncol. 126 (2012) 466-473.

[9] V.H. Jongen, J.M. Briet, R.A. de Jong, E. Joppe, K.A. ten Hoor, H.M. Boezen, et al., Aromatase, cyclooxygenase 2, HER-2/neu, and p53 as prognostic factors in endometrioid endometrial cancer, Int. J. Gynecol. Cancer 19 (2009) 670-676.

[10] D.E. Cohn, W.L. Frankel, K.E. Resnick, V.L. Zanagnolo, L.J. Copeland, H. Hampel, et al., Improved survival with an intact DNA mismatch repair system in endometrial cancer, Obstet. Gynecol. 108 (2006) 1208-1215.

[11] I.M. Stefansson, H.B. Salvesen, H. Immervoll, L.A. Akslen, Prognostic impact of histological grade and vascular invasion compared with tumour cell proliferation in endometrial carcinoma of endometrioid type, Histopathology 44 (2004) 472-479.

[12] A.D. Santin, HER2/neu overexpression: has the Achilles' heel of uterine serous papillary carcinoma been exposed, Gynecol. Oncol. 88 (2003) 263-265.

[13] T. Bosse, R.A. Nout, E. Stelloo, E. Dreef, H.W. Nijman, I.M. Jürgenliemk-Schulz, et al., L1 cell adhesion molecule is a strong predictor for distant recurrence and overall survival in early stage endometrial cancer: pooled PORTEC trial results, Eur. J. Cancer 50 (2014) 2602-2610.

[14] A. Pasanen, T. Tuomi, J. Isola, Staff S, R. Bützow, M. Loukovaara, L1 cell adhesion molecule as a predictor of disease-specific survival and patterns of relapse in endometrial cancer, Int. J. Gynecol. Cancer 26 (2016) 1465-1471.

[15] I.C. Van Gool, E. Stelloo, R.A. Nout, H.W. Nijman, R.J. Edmondson, D.N. Church, et al., Prognostic significance of L1CAM expression and its association with mutant p53 expression in high-risk endometrial cancer, Mod. Pathol. 29 (2016) 174-181.

[16] T.H. Dellinger, D.D. Smith, C. Ouyang, C.D. Warden, J.C. Williams, E.S. Han, L1CAM is an independent predictor of poor survival in endometrial cancer - an analysis of the cancer genome atlas (TCGA), Gynecol. Oncol. 141 (2016) 336-340.

[17] Y.P. Geels, J.M. Pijnenborg, B.B. Gordon, M. Fogel, P. Altevogt, R. Masadah, et al., L1CAM expression is related to non-Endometrioid histology, and prognostic for poor outcome in Endometrioid endometrial carcinoma, Pathol. Oncol. Res. 22 (2016) 863-868.

[18] Y.P. Geels, L.J. van der Putten, A.A. van Tilborg, I. Lurkin, E.C. Zwarthoff, J.M. Pijnenborg, et al., Immunohistochemical and genetic profiles of endometrioid endometrial carcinoma arising from atrophic endometrium, Gynecol. Oncol. 137 (2015) 245-251.

[19] P.H. Edqvist, J. Huvila, B. Forsström, L. Talve, O. Carpén, H.B. Salvesen, et al., Loss of ASRGL1 expression is an independent biomarker for disease-specific survival in endometrioid endometrial carcinoma, Gynecol. Oncol. 137 (2015) 529-537.

[20] T. Fonnes, H.F. Berg, T. Bredholt, P.D. Edqvist, K. Sortland, A. Berg, et al., Asparaginase-like protein 1 is an independent prognostic marker in primary endometrial cancer, and is frequently lost in metastatic lesions, Gynecol. Oncol. 148 (2018) 197-203.

[21] T.A. O'Mara, M. Zhao, A.B. Spurdle, Meta-analysis of gene expression studies in endometrial cancer identifies gene expression profiles associated with aggressive disease and patient outcome, Sci. Rep. 6 (2016), 36677.

[22] Cancer Genome Atlas Research Network, C. Kandoth, N. Schultz, A.D. Cherniack, R. Akbani, Y. Liu, et al., Integrated genomic characterization of endometrial carcinoma, Nature 497 (2013) 67-73.

[23] W. Creasman, Revised FIGO staging for carcinoma of the endometrium, Int. J. Gynaecol. Obstet. 105 (2009) 109.

[24] R.J. Zaino, R.J. Kurman, K.L. Diana, C.P. Morrow, The utility of the revised International Federation of Gynecology and Obstetrics histologic grading of endometrial adenocarcinoma using a defined nuclear grading system. A gynecologic oncology group study, Cancer 75 (1995) 81-86.

[25] C. Kampf, I. Olsson, U. Ryberg, E. Sjostedt, F. Ponten, Production of tissue microarrays, immunohistochemistry staining and digitalization within the human protein atlas, J. Vis. Exp. (2012)https://doi.org/10.3791/3620.

[26] M. Uhlén, L. Fagerberg, B.M. Hallström, C. Lindskog, P. Oksvold, A. Mardinoglu, et al., Proteomics. Tissue-based map of the human proteome, Science 347 (2015), 1260419. 
[27] R.A. Walker, Quantification of immunohistochemistry-issues concerning methods, utility and semiquantitative assessment I, Histopathology 49 (2006) 406-410.

[28] J. Friedman, T. Hastie, R. Tibshirani, Regularization paths for generalized linear models via coordinate descent, J. Stat. Softw. 33 (2010) 1-22.

[29] N. Simon, J. Friedman, T. Hastie, R. Tibshirani, Regularization paths for Cox's proportional hazards model via coordinate descent, J. Stat. Softw. 39 (2011) 1-13.

[30] TCGA Research Network. Retrieved 30.5.2017, http://cancergenome.nih.gov/.

[31] A. Talhouk, M.K. McConechy, S. Leung, W. Yang, A. Lum, J. Senz, et al., Confirmation of ProMisE: a simple, genomics-based clinical classifier for endometrial cancer, Cancer 123 (2017) 802-813.

[32] L. Helpman, R. Kupets, A. Covens, R.S. Saad, M.A. Khalifa, N. Ismiil, et al., Assessment of endometrial sampling as a predictor of final surgical pathology in endometrial cancer, Br. J. Cancer 110 (2014) 609-615.

[33] A.K. Folkins, N.S. Nevadunsky, A. Saleemuddin, E.A. Jarboe, M.G. Muto, C.M. Feltmate, et al., Evaluation of vascular space involvement in endometrial adenocarcinomas: laparoscopic vs abdominal hysterectomies, Mod. Pathol. 23 (2010) 1073-1079.

[34] S. Bendifallah, G. Canlorbe, P. Collinet, E. Arsène, F. Huguet, C. Coutant, et al., Just how accurate are the major risk stratification systems for early-stage endometrial cancer, Br. J. Cancer 112 (2015) 793-801.

[35] H.M. Werner, J. Trovik, J. Marcickiewicz, S. Tingulstad, Staff AC, M.E. Engh, et al., A discordant histological risk classification in preoperative and operative biopsy in endometrial cancer is reflected in metastatic risk and prognosis, Eur. J. Cancer 49 (2013) 625-632.
[36] L.J. van der Putten, N.C. Visser, K. van de Vijver, M. Santacana, P. Bronsert, J. Bulten, et al., L1CAM expression in endometrial carcinomas: an ENITEC collaboration study, Br. J. Cancer 115 (2016) 716-724.

[37] C.B. Gilks, E. Oliva, R.A. Soslow, Poor interobserver reproducibility in the diagnosis of high-grade endometrial carcinoma, Am. J. Surg. Pathol. 37 (2013) 874-881.

[38] L.N. Hoang, M.A. Kinloch, J.M. Leo, K. Grondin, C.H. Lee, C. Ewanowich, et al., Interobserver agreement in endometrial carcinoma histotype diagnosis varies depending on the cancer genome atlas (TCGA)-based molecular subgroup, Am. J. Surg. Pathol 41 (2017) 245-252.

[39] J.R. Cantor, E.M. Stone, L. Chantranupong, G. Georgiou, The human asparaginase-like protein 1 hASRGL1 is an Ntn hydrolase with beta-aspartyl peptidase activity, Biochemistry 48 (2009) 11026-11031.

[40] S.R.V. Knott, E. Wagenblast, S. Khan, S.Y. Kim, M. Soto, M. Wagner, et al., Asparagine bioavailability governs metastasis in a model of breast cancer, Nature 554 (2018) 378-381.

[41] Y.R. Hussein, R. Broaddus, B. Weigelt, D.A. Levine, R.A. Soslow, The genomic heterogeneity of FIGO grade 3 endometrioid carcinoma impacts diagnostic accuracy and reproducibility, Int. J. Gynecol. Pathol. 35 (2016) 16-24.

[42] A. Talhouk, L.N. Hoang, M.K. McConechy, Q. Nakonechny, J. Leo, A. Cheng, et al., Molecular classification of endometrial carcinoma on diagnostic specimens is highly concordant with final hysterectomy: earlier prognostic information to guide treatment, Gynecol. Oncol. 143 (2016) 46-53. 